\title{
Esophageal Intramucosal Adenocarcinoma
}

National Cancer Institute

\section{Source}

National Cancer Institute. Esophageal Intramucosal Adenocarcinoma. NCI Thesaurus.

Code C161906.

Adenocarcinoma that arises from the esophagus and is confined entirely to the mucosa. 